Research Article

\title{
Relationship between Periodontitis and Glaucoma: A Cross-Sectional Study
}

\author{
Soo Hwan Byun, ${ }^{1,2}$ Dae Myoung Yoo, ${ }^{3}$ Minwook Chang, ${ }^{4}$ Hyo Geun Choi $\mathbb{D}^{2,3,5}$ \\ and Seok Jin Hong $\mathbb{D D}^{2,6,7}$ \\ ${ }^{1}$ Department of Oral \& Maxillofacial Surgery, Dentistry, Sacred Heart Hospital, Hallym University, College of Medicine, \\ 14068 Anyang, Republic of Korea \\ ${ }^{2}$ Research Centre of Clinical Dentistry, Hallym University, Clinical Dentistry Graduate School, 24252 Chuncheon, \\ Republic of Korea \\ ${ }^{3}$ Hallym Data Science Laboratory, Hallym University College of Medicine, 14068 Anyang, Republic of Korea \\ ${ }^{4}$ Department of Ophthalmology, Dongguk University Ilsan Hospital, 10326 Goyang, Republic of Korea \\ ${ }^{5}$ Department of Otorhinolaryngology-Head \& Neck Surgery, Sacred Heart Hospital, Hallym University, College of Medicine, \\ 14068 Anyang, Republic of Korea \\ ${ }^{6}$ Department of Otorhinolaryngology-Head \& Neck Surgery, Dongtan Sacred Heart Hospital, Hallym University, \\ College of Medicine, 18450 Dongtan, Republic of Korea \\ ${ }^{7}$ Allergy and Clinical Immunology Research Center, Hallym University College of Medicine, 18450 Dongtan, Republic of Korea
}

Correspondence should be addressed to Hyo Geun Choi; pupen@naver.com and Seok Jin Hong; enthsj@hanmail.net

Received 19 June 2020; Revised 15 November 2020; Accepted 18 November 2020; Published 2 December 2020

Academic Editor: Nra Szentmry

Copyright (c) 2020 Soo Hwan Byun et al. This is an open access article distributed under the Creative Commons Attribution License, which permits unrestricted use, distribution, and reproduction in any medium, provided the original work is properly cited.

\begin{abstract}
The objective of this cross-sectional study was to evaluate the association between periodontitis and glaucoma. This prospective cohort study used epidemiological data from the Korean Genome and Epidemiology Study performed between 2004 and 2016. Among 173,209 participants, 9572 patients with periodontitis and 115,332 controls (nonperiodontitis) were selected. We analysed the history of glaucoma in periodontitis and control participants. The participants were interviewed regarding their history of hypertension, diabetes mellitus, hyperlipidaemia, periodontitis, glaucoma, smoking, and alcohol consumption by trained interviewers. A logistic regression model was created to analyse the odds ratio of having a history of glaucoma among patients with periodontitis. Two-tailed analyses using chi-square and independent $t$-tests were used for statistical analysis. The adjusted odds ratio of periodontitis as a risk factor for glaucoma was 3.44 (95\% confidence interval $=2.99-3.97, p<0.001)$. This study demonstrated that glaucoma was associated with periodontitis.
\end{abstract}

\section{Introduction}

Periodontitis is one of the most prevalent diseases and manifests as the destruction of the periodontal tissues $[1,2]$. The presence of pathogenic bacteria, often known as the "periopathogens," in the gingival sulcus is the principal etiology of periodontitis [3]. Inflammatory response, poor oral hygiene, malnutrition, and smoking are essential etiological factors of chronic periodontitis, which are mediated by proteolytic enzymes and lipopolysaccharides [4]. In addition, environmental and genetic factors mediate the immune response of the host [5]. The characteristics of periodontitis include periodontal inflammation, bone and periodontal attachment loss leading to tooth mobility and formation of periodontal pockets, gingival inflammation, gingival bleeding, and/or gingival recession [6]. A previous study reported that the prevalence of periodontitis in the United States is approximately 60 to $70 \%$ in older adults [7]. Its increased prevalence prompted various large population studies designed to identify the association between periodontitis and systemic diseases common in the old age. 
Glaucoma is a chronic degenerative ophthalmic disease caused by damage to the optic nerve and often results in loss of vision. It is an optic neuropathy and is the second-leading cause of blindness worldwide [8]. Based on its association with intraocular pressure, glaucoma is divided into subtypes according to the condition of the iridocorneal drainage angle at the site of aqueous humor egress from the eye [9].

The most common type is open-angle glaucoma, which develops slowly, is painless, and often results in blindness if not appropriately managed. Open-angle glaucoma exhibits an iridocorneal angle favouring unobstructed drainage of fluid within the eyes, and closed-angle glaucoma presents progressive closure of the drainage angle because of the sudden or gradual forward bowing of the peripheral iris. The symptoms of sudden bowing include blurred vision, severe pain in the eye, redness of the eye, a middilated pupil, and nausea. Decreased vision loss due to glaucoma is irreversible and is caused by the loss of axons in the ganglion cell layer owing to the thinning neuroretinal rim of the optic nerve [9]. Glaucoma is an ocular disorder that involves the loss of retinal ganglion cells and related axons. There are various hypotheses concerning the cause of death in retinal ganglion cells. In the past decade, glaucoma has been defined by high intraocular pressure; however, recent studies have shown that elevated intraocular pressure is the most important risk factor for glaucoma, but it does not define the disease. Other factors such as genetics, age, blood flow, and systemic diseases are suggested as potential risk factors for the progressive retinal ganglion cell death observed in glaucoma $[10,11]$. Approximately $3 \%$ of the world's population aged $>40$ years is affected by glaucoma [8]. Age has been the factor most frequently associated with glaucoma [8]; other factors include ethnicity, hypertension, genetics, diabetes, and trauma [12].

The prevalence of both periodontitis and glaucoma increases with age and affected by systemic factors such as diabetes, smoking, alcohol consumption, income, and body mass index (BMI). Few studies have analysed the association between periodontitis and ophthalmic diseases. Hence, this cross-sectional study aimed to evaluate the association between periodontitis and glaucoma, after adjusting for systemic factors, using the Korean Genome and Epidemiology Study (KoGES) data.

\section{Materials and Methods}

2.1. Study Population and Data Collection. This prospective cohort study analysed the data from the KoGES performed from 2004 to 2016, which comprised base data from 2004 to 2013 and follow-up data from 2012 to 2016. The data have been described in detail in a previous study [13]. From the KoGES consortium, we used the KoGES health examinee (HEXA) data of urban residents $\geq 40$ years of age. The ethics committee of the Hallym University (2019-02-020) approved the use of these data. The requirement for written informed consent was waived by the Institutional Review Board. The study was conducted in full accordance with ethical principles, including the rules of the Declaration of Helsinki of 1975, revised in 2013.
2.2. Participant Selection. From the 173,209 participants of the KoGES, those who lacked the data pertaining to height or weight ( $n=698)$, smoking history $(n=494)$, habitual alcohol consumption $(n=1463)$, hypertension, diabetes mellitus, hyperlipidaemia $(n=125)$, periodontitis $(n=33,849)$, and glaucoma history $(n=11,676)$ were excluded. Several participants with a history of periodontitis were not surveyed from 2004 to 2006, and those with glaucoma were not surveyed in 2004 and 2008. All these participants were excluded. Finally, 9572 participants with periodontitis and 115,332 controls (no history of periodontitis) were included in the study. The history of glaucoma was analysed in the periodontitis and control participants.

2.3. Survey. The participants were questioned about their history of hypertension, diabetes mellitus, hyperlipidaemia, periodontitis, and glaucoma by trained interviewers. The questions were structured as follows: "Have you ever had a diagnosis of periodontitis?" and "Do you have any history of glaucoma?" BMI of the participants was calculated in $\mathrm{kg} / \mathrm{m}^{2}$ using the data obtained from the general health examination. Participants were categorized as nonsmokers $(<100$ cigarettes in entire life), past smokers (stopped smoking more than one year ago), and current smokers based on their smoking history and as nondrinkers, past drinkers, and current drinkers based on their history of alcohol consumption. They were categorized into nonrespondent, lowincome $\quad(<\sim 2000$ per month $)$ middle-income ( $\sim 2000-\$ 3999$ per month), and high-income ( $\sim \$ 4000$ per month) groups based on their household income. Additionally, participants were categorized according to their BMI as underweight for $\mathrm{BMI}<18.5 \mathrm{~kg} / \mathrm{m}^{2}$, normal weight for BMI $18.5-23 \mathrm{~kg} / \mathrm{m}^{2}$, overweight for BMI $23-25 \mathrm{~kg} / \mathrm{m}^{2}$, and obese for BMI $\geq 25 \mathrm{~kg} / \mathrm{m}^{2}$ [14].

2.4. Statistical Analyses. A chi-square test was used to compare categorical variables such as sex, income group, smoking, alcohol consumption, and history of hypertension, diabetes mellitus, hyperlipidaemia, and glaucoma. An independent $t$-test was used to compare continuous variables such as age, BMI, and nutritional status.

A logistic regression model was created to analyse the odds ratio (OR) of having a history of glaucoma among patients with periodontitis. Crude and adjusted models (age, sex, income group, BMI, smoking, alcohol consumption, hypertension, diabetes mellitus, and hyperlipidaemia) were used. For subgroup analyses according to age and sex, the median age ( $\leq 52$ years old and $>52$ years old) was considered as the dividing point.

Subgroup analyses according to medical history of hypertension, diabetes mellitus, and hyperlipidaemia were performed, and a separate subgroup analysis was performed for BMI. As the proportion of underweight participants was $\sim 1.8 \%$, they were merged into the normal-weight group.

Two-tailed analyses were conducted, and a $p$ value less than 0.05 was considered to indicate statistical significance. The results were statistically analysed using SPSS v. 24.0 (IBM, Armonk, NY, USA). 


\section{Results}

In the baseline data, $1.4 \%(134 / 9,572)$ of the periodontitis participants had glaucoma, and in the follow-up, 0.6\% (53/ $9,572)$ of these participants had developed glaucoma. In contrast, $0.6 \%(664 / 115,332)$ of the nonperiodontitis participants had glaucoma in the first survey, and in the follow-up, $0.3 \%(3990 / 115,332)$ of the nonperiodontitis participants had developed glaucoma. The general characteristics of the periodontitis and control participants were significantly different (Table 1). The adjusted OR (aOR) of periodontitis as a risk factor for glaucoma was 3.44 (95\% confidence interval $(\mathrm{CI})=2.99-3.97, p<0.001$, Table 2). Subgroup analyses according to age and sex showed aORs of 7.17 (95\% CI $=4.11-12.51)$ in $\leq 52$-year-old men; 8.04 (95\% CI $=4.78-13.51)$ in $\leq 52$-year-old women; $3.31(95 \% \mathrm{CI}=2.62-4.17)$ in $>52$-year-old men; and 2.96 $(95 \% \mathrm{CI}=2.43-3.61)$ in $>52$-year-old women.

The aOR of periodontitis as a risk factor for glaucoma was $2.05(95 \% \mathrm{CI}=1.61-2.61)$ in participants with hypertension, while it was $1.67(95 \% \mathrm{CI}=1.35-2.06)$ in participants without hypertension (Table 3). It was 2.00 (95\% $\mathrm{CI}=1.44-2.78)$ in participants with diabetes mellitus and $1.77(95 \% \mathrm{CI}=1.47-2.12)$ in participants without diabetes mellitus, and it was $1.85(95 \% \mathrm{CI}=1.41-2.43)$ in participants with hyperlipidaemia and $1.80(95 \% \mathrm{CI}=1.48-2.19)$ in participants without hyperlipidaemia.

The aOR of periodontitis as a risk factor for glaucoma was $1.50(95 \% \mathrm{CI}=1.12-2.01)$ in underweight or normalweight participants, $1.73(95 \% \mathrm{CI}=1.29-2.32)$ in overweight participants, and $2.23(95 \% \mathrm{CI}=1.74-2.86)$ in obese participants (Table 4).

\section{Discussion}

Similar to other systems, deteriorating ophthalmic function is related to age and, hence, is more prevalent in the older population [15]. When the visual field and visual acuity are compromised, they not only affect the individual's ability to see but also affect the function of other systems [9]. Similarly, previous studies on periodontitis showed that aging leads to increased osteoclastic activity, enhancing the susceptibility to periodontitis [16-18]. Aging-related association of ophthalmic disease and periodontitis seems obvious. However, this study attempted to analyse the association between periodontitis and glaucoma, using other possible predictive factors. This study revealed that glaucoma and periodontitis were significantly associated at all ages and in both sexes even after adjustment for several confounding factors including age, diabetes, hypertension, BMI, income, smoking, and alcohol consumption. Periodontitis and glaucoma are associated with chronic inflammation and innate immunity; innate immunity is known to play a role in various chronic inflammatory diseases [19]. Periodontitis stimulates an inflammatory response and induces innate immunity response through the activation of toll-like receptors (TLRs) [20]. Chronic exposure to bacteria, viruses, fungal spores, and environmental
TABLE 1: General characteristics of participants.

\begin{tabular}{|c|c|c|c|}
\hline \multirow{2}{*}{ Characteristics } & \multicolumn{2}{|c|}{ Total participants } & \multirow{2}{*}{$p$ value } \\
\hline & Periodontitis & Control & \\
\hline Age (mean, SD, $y$ ) & $54.9(7.9)$ & $53.0(8.3)$ & $<0.001^{*}$ \\
\hline $\operatorname{Sex}(n, \%)$ & & & $<0.001^{*}$ \\
\hline Men & $3679(38.4)$ & $39,727(34.4)$ & \\
\hline Women & $5893(61.6)$ & $75,605(65.6)$ & \\
\hline $\mathrm{BMI}\left(\right.$ mean, $\left.\mathrm{SD}, \mathrm{kg} / \mathrm{m}^{2}\right)$ & $24.0(2.9)$ & $23.9(2.9)$ & $<0.001^{*}$ \\
\hline Income $(n, \%)$ & & & $<0.001^{*}$ \\
\hline Missing, no response & $742(7.8)$ & $10,173(8.8)$ & \\
\hline Lowest & $3294(34.4)$ & $32,701(28.4)$ & \\
\hline Middle & $3556(37.2)$ & $45,446(39.4)$ & \\
\hline Highest & $1980(20.7)$ & $27,012(23.4)$ & \\
\hline Hypertension & $2703(28.2)$ & $26,492(23.0)$ & $<0.001^{*}$ \\
\hline Diabetes mellitus & $1153(12.0)$ & $9254(8.0)$ & $<0.001^{*}$ \\
\hline Hyperlipidaemia & $2171(22.7)$ & $17,046(14.8)$ & $<0.001^{*}$ \\
\hline Smoking status $(n, \%)$ & & & $<0.001^{*}$ \\
\hline Nonsmoker & $6428(67.2)$ & $83,955(72.8)$ & \\
\hline Past smoker & $1717(17.9)$ & $17,270(15.0)$ & \\
\hline Current smoker & $1427(14.9)$ & $14,107(12.2)$ & \\
\hline $\begin{array}{l}\text { Alcohol } \\
\text { consumption (n, \%) }\end{array}$ & & & $<0.001^{*}$ \\
\hline Nondrinker & $4594(48.0)$ & $58,888(51.1)$ & \\
\hline Past drinker & $461(4.8)$ & $4282(3.7)$ & \\
\hline Current drinker & $4517(47.2)$ & $52,162(45.2)$ & \\
\hline Glaucoma $(n, \%)$ & $187(2.0)$ & $1054(0.9)$ & $<0.001^{*}$ \\
\hline
\end{tabular}

${ }^{*}$ Independent $t$-test or chi-square test. Significance at $p<0.05$.

stressors can cause oral epithelial cells to secrete cytokines (IL-1, IL-6, IL-17, and TNF- $\alpha$ ). These cytokines activate several inflammatory pathways and recruit dedicated immune cells (macrophages, dendritic cells, eosinophils, neutrophils, T cells, and natural-killer cells) that could play significant roles in the pathogenesis of chronic periodontitis [21,22]. Glaucoma is characterized by elevated intraocular pressure; however, it is more accurately defined as a neuroinflammatory and neurodegenerative disease that results in the degeneration of the retinal ganglion cells [23]. In glaucoma, elevated intraocular pressure triggers an innate immune response, which usually involves resident immune cells such as microglia as well as the infiltration of macrophages and monocytes [24]. The neuroinflammatory response activated by microglia is believed to play a leading role in the pathophysiology of glaucoma. Activated microglia induce secretion of proinflammatory cytokines (IL-1, IL-6, and TNF- $\alpha$ ) and trigger innate immune responses through TLRs [25-27]. Inflammation and innate immunity are essential for understanding the link between periodontitis and glaucoma. In addition, oxidative stress is a potential common pathophysiology for the two conditions. Oxidative stress is defined as an imbalance between the antioxidant mechanism and production of highly reactive molecular species such as reactive oxygen/ nitrogen species (ROS/RNS) $[28,29]$. Previous studies have revealed that patients with chronic periodontitis exhibit lower antioxidant capacity than controls, and bacteria may be related to oxidative stress in patients with periodontitis [30-35]. The pathophysiology of oxidative 
TABle 2: Crude and adjusted odds ratios (95\% confidence interval) of periodontitis as a risk factor for glaucoma.

\begin{tabular}{|c|c|c|c|c|}
\hline \multirow{2}{*}{ Characteristics } & \multicolumn{4}{|c|}{ Odds ratios for glaucoma } \\
\hline & Crude & $p$ value & Adjusted $^{\dagger}$ & $p$ value \\
\hline \multicolumn{5}{|c|}{ Total participants $(n=124,904)$} \\
\hline Periodontitis & $5.96(5.23-6.79)$ & $<0.001^{*}$ & $3.44(2.99-3.97)$ & $<0.001^{*}$ \\
\hline Control & 1.00 & & 1.00 & \\
\hline \multicolumn{5}{|c|}{ Age $\leq 52$ years, men $(n=19,428)$} \\
\hline Periodontitis & $8.61(5.04-14.69)$ & $<0.001^{*}$ & $7.17(4.11-12.51)$ & $<0.001^{*}$ \\
\hline Control & 1.00 & & 1.00 & \\
\hline \multicolumn{5}{|c|}{ Age $\leq 52$ years, women $(n=42,000)$} \\
\hline Periodontitis & $10.52(6.39-17.32)$ & $<0.001^{*}$ & $8.04(4.78-13.51)$ & $<0.001^{*}$ \\
\hline Control & 1.00 & & 1.00 & \\
\hline \multicolumn{5}{|c|}{ Age $>52$ years, men $(n=23,978)$} \\
\hline Periodontitis & $4.05(3.25-5.06)$ & $<0.001^{*}$ & $3.31(2.62-4.17)$ & $<0.001^{*}$ \\
\hline Control & 1.00 & & 1.00 & \\
\hline \multicolumn{5}{|c|}{ Age $>52$ years, women $(n=39,498)$} \\
\hline Periodontitis & $4.07(3.38-4.89)$ & $<0.001^{*}$ & $2.96(2.43-3.61)$ & $<0.001^{*}$ \\
\hline Control & 1.00 & & 1.00 & \\
\hline
\end{tabular}

${ }^{*}$ Logistic regression model; significance at $p<0.05 .{ }^{\dagger}$ Models adjusted for age, sex, income group, BMI, smoking, alcohol consumption, hypertension, diabetes mellitus, and hyperlipidaemia.

TABLE 3: Crude and adjusted odds ratios (95\% confidence interval) of periodontitis as a risk factor for glaucoma according to their past medical histories of hypertension, diabetes mellitus, and hyperlipidaemia.

\begin{tabular}{|c|c|c|c|c|}
\hline \multirow{2}{*}{ Characteristics } & \multicolumn{4}{|c|}{ Odds ratios for glaucoma } \\
\hline & Crude & $p$ value & Adjusted $^{\dagger}$ & $p$ value \\
\hline \multicolumn{5}{|c|}{ Participants with hypertension $(n=29,195)$} \\
\hline Periodontitis & $2.24(1.77-2.84)$ & $<0.001^{*}$ & $2.05(1.61-2.61)$ & $<0.001^{*}$ \\
\hline Control & 1.00 & & 1.00 & \\
\hline \multicolumn{5}{|c|}{ Participants without hypertension $(n=95,798)$} \\
\hline Periodontitis & $1.97(1.59-2.42)$ & $<0.001^{*}$ & $1.67(1.35-2.06)$ & $<0.001^{*}$ \\
\hline Control & 1.00 & & 1.00 & \\
\hline \multicolumn{5}{|c|}{ Participants with diabetes mellitus $(n=10,407)$} \\
\hline Periodontitis & $2.08(1.50-2.89)$ & $<0.001^{*}$ & $2.00(1.44-2.78)$ & $<0.001^{*}$ \\
\hline Control & 1.00 & & 1.00 & \\
\hline \multicolumn{5}{|c|}{ Participants without diabetes mellitus $(n=114,497)$} \\
\hline Periodontitis & $2.05(1.71-2.45)$ & $<0.001^{*}$ & $1.77(1.47-2.12)$ & $<0.001^{*}$ \\
\hline Control & 1.00 & & 1.00 & \\
\hline \multicolumn{5}{|c|}{ Participants with hyperlipidaemia $(n=19,217)$} \\
\hline Periodontitis & $1.99(1.52-2.60)$ & $<0.001^{*}$ & $1.85(1.41-2.43)$ & $<0.001^{*}$ \\
\hline Control & 1.00 & & 1.00 & \\
\hline \multicolumn{5}{|c|}{ Participants without hyperlipidaemia $(n=105,687)$} \\
\hline Periodontitis & $2.04(1.68-2.48)$ & $<0.001^{*}$ & $1.80(1.48-2.19)$ & $<0.001^{*}$ \\
\hline Control & 1.00 & & 1.00 & \\
\hline
\end{tabular}

${ }^{*}$ Logistic regression model; significance at $p<0.05 .{ }^{\dagger}$ Models adjusted for age, sex, income group, BMI, smoking, alcohol consumption, hypertension, diabetes mellitus, and hyperlipidaemia.

Table 4: Crude and adjusted odds ratios (95\% confidence interval) of periodontitis for glaucoma according to body mass index.

\begin{tabular}{|c|c|c|c|c|}
\hline \multirow{2}{*}{ Characteristics } & \multicolumn{4}{|c|}{ Odds ratios for glaucoma } \\
\hline & Crude & $p$ value & Adjusted $^{\dagger}$ & $p$ value \\
\hline \multicolumn{5}{|c|}{ Participants with BMI $<23$ (underweight or normal, $n=49,900$ ) } \\
\hline Periodontitis & $1.83(1.38-2.44)$ & $<0.001^{*}$ & $1.50(1.12-2.01)$ & $0.006^{*}$ \\
\hline Control & 1.00 & & 1.00 & \\
\hline \multicolumn{5}{|c|}{ Participants with $\mathrm{BMI} \geq 23$ and $<25$ (overweight, $n=34,604$ ) } \\
\hline Periodontitis & $2.01(1.50-2.68)$ & $<0.001^{*}$ & $1.73(1.29-2.32)$ & $<0.001^{*}$ \\
\hline Control & 1.00 & & 1.00 & \\
\hline \multicolumn{5}{|c|}{ Participants with $\mathrm{BMI} \geq 25$ (obese, $n=40,400$ ) } \\
\hline Periodontitis & $2.59(2.02-3.31)$ & $<0.001^{*}$ & $2.23(1.74-2.86)$ & $<0.001^{*}$ \\
\hline Control & 1.00 & & 1.00 & \\
\hline
\end{tabular}

${ }^{*}$ Logistic regression model; significance at $p<0.05 .{ }^{\dagger}$ Models adjusted for age, sex, income group, BMI, smoking, alcohol consumption, hypertension, diabetes mellitus, and hyperlipidaemia. 
stress in periodontitis has been considered for decades; however, it has not been completely understood [36, 37]. Some studies have reported that the number of leukocytes is decreased in periodontitis patients and that they also exhibit low oxidative activity [38]. Other studies have found higher numbers of free radicals in the leukocytes of periodontitis patients [39]. These contradictory findings could be related to the dynamic pathophysiology of periodontitis and could explain the different types of periodontitis [40]. The mechanism of oxidative stress is ambiguous, and antioxidants can affect various processes that are not directly related to free radical generation and/or action [41]. Several studies have revealed that oxidative stress is closely related to periodontitis, but the mechanism of oxidative stress in the pathophysiology of periodontitis is not clear [42]. Oxidative stress in periodontitis is assumed to be related to the pathogenesis of various diseases. In fact, most inflammatory diseases induce oxidative stress. However, few studies have analysed the oxidative stress in systemic diseases with regard to periodontal status $[43,44]$. Nguyen et al. investigated the association between acute coronary syndrome and chronic periodontitis [43]. Another study reported that both rheumatoid arthritis and chronic periodontitis showed definitive higher levels of oxidative stress markers in plasma and lower antioxidant capacity [45]. Oxidative stress has been associated with several ophthalmic diseases such as glaucoma, ocular surface disorders, and diabetic retinopathy [46-48]. Oxidative damage was also evident in the DNA of primary open-angle glaucoma patients [49]. It has been revealed that primary open-angle glaucoma patients are genetically predisposed to ROS-induced damage [49]. Oxidative stress influences the trabecular meshwork, Schlemm's canal, retinal ganglion cells, and optic nerve fibres [48]. Oxidative stress has also been linked to primary open-angle glaucoma by increasing the flow resistance in the anterior chamber of the eye. However, further investigations are needed to identify the exact signalling pathways regulated by ROS in ophthalmic diseases. In order to evaluate the effect of obesity, hypertension, diabetes, and hyperlipidaemia on the association between periodontitis and glaucoma, additional evaluations were performed with decreasing adjustments for obesity, hypertension, diabetes, and hyperlipidaemia (Tables 3 and 4). As a result, a possibility was evident that the association between periodontitis and glaucoma would be increased by presence of obesity, hypertension, diabetes, and hyperlipidaemia. Periodontitis has also been associated with insulin resistance and metabolic syndromes including hypertension and diabetes, which are again characterized by oxidative stress [50]. Some researchers have reported that primary open-angle glaucoma is positively associated with hypertension and diabetes mellitus $[51,52]$. Many studies have shown that proinflammatory cytokines such as IL-1, IL-6, and TNF- $\alpha$ and immune responses could serve as a link between chronic periodontitis and other chronic diseases $[22,50,53]$. All these factors seem to act as a common link to explain the relationships among periodontitis, hypertension, diabetes, hyperlipidaemia, and glaucoma [50, 54]. With the increase in BMI, the association between glaucoma and periodontitis significantly increased (Table 4 ). This result suggests that glaucoma is linked to periodontitis through factors of insulin resistance and oxidative stress, and a high possibility of glaucoma may be evident in obese periodontitis patients. These additional evaluations included various adjustment methods mentioned above and showed the effects of obesity, hypertension, diabetes, and hyperlipidaemia on the association between periodontitis and glaucoma. This study used data from a large population. Nevertheless, the study has some limitations. First, the data were collected using a questionnaire survey. Hence, the data used in this study may not be completely accurate. Data on confounding factors such as diabetes and inflammation conditions could be incorrect due to the subjective nature of the survey, which could have influenced the study findings. Second, it was infeasible to adjust for all factors that were not included in the data. However, we attempted to adjust the maximum number of factors to minimize surveillance bias. Third, the validity of the questionnaire in terms of frequencies of alcohol consumption and smoking is questionable due to social desirability bias. Periodontitis can also progress unnoticed until the teeth becomes mobile or useless; hence, participants might not have been able to accurately diagnose periodontitis. Lastly, the mechanism of the relationship between periodontitis and glaucoma cannot be clarified through the results of this study alone, and further long-term studies are required to validate this association. Despite these limitations, this study provided critical information about periodontitis and glaucoma. First, our study was performed in a large sample of the Korean population. Second, we adjusted the results for the factors of age, sex, obesity, income, hypertension, hyperlipidaemia, smoking, diabetes, and alcohol consumption to evaluate the independent relationship between periodontitis and glaucoma. If these factors were not adequately adjusted, they could have influenced the association of periodontitis with glaucoma. The adjustments helped in obtaining definitive and valid results, without any bias.

\section{Conclusions}

This study showed the association between glaucoma and periodontitis and suggested a possible mechanism for this association. However, further studies would be needed to overcome the limitations of this study.

\section{Data Availability}

Access to data is restricted by the Korean government policy.

\section{Conflicts of Interest}

The authors declare that there are no conflicts of interest regarding the publication of this paper.

\section{Authors' Contributions}

Hyo Geun Choi and Seok Jin Hong equally contributed to this study. 


\section{Acknowledgments}

This work was supported in part by a research grant (NRF2018-R1D1A1A0-2085328) from the National Research Foundation of Korea (NRF). This study was supported by the National Research Foundation of Korea (NRF) grant funded by the Korean Government Ministry of Science and ICT (no. 2017R1C1B5076558). This study was also supported by the Hallym University Research Fund (HURF).

\section{References}

[1] Global Burden of Disease Study, "Injury I and Prevalence C 2016 Global, regional, and national incidence, prevalence, and years lived with disability for 310 diseases and injuries, 1990-2015: a systematic analysis for the Global Burden of Disease Study," Lancet, vol. 388, pp. 1545-1602, 2015.

[2] N. J. Kassebaum, A. G. C. Smith, E. Bernabé et al., "Regional, and national prevalence, incidence, and disability-adjusted life years for oral conditions for 195 countries, 1990-2015: a systematic analysis for the global burden of diseases, injuries, and risk factors," Journal of Dental Research, vol. 96, no. 4, pp. 380-387.

[3] S. S. BenzianAsayesh, "Microbiology of periodontal diseasepresent status and future considerations," Journal of Periodontology, vol. 48, no. 9, pp. 497-504, 1977.

[4] M. L. Laine, W. Crielaard, and B. G. Loos, "Genetic susceptibility to periodontitis," Periodontology 2000, vol. 58, no. 1, pp. 37-68, 2012.

[5] K. S. Kornman, R. C. Page, and M. S. Tonetti, "The host response to the microbial challenge in periodontitis: assembling the players," Periodontology 2000, vol. 14, no. 1, pp. 33-53, 1997.

[6] G. C. Armitage, "Periodontal diagnoses and classification of periodontal diseases," Periodontology 2000, vol. 34, no. 1, pp. 9-21, 2004.

[7] P. I. Eke, L. Wei, W. S. Borgnakke et al., "Periodontitis prevalence in adults $\geq 65$ years of age, in the USA," Periodontology 2000, vol. 72, no. 1, pp. 76-95, 2016.

[8] H. A. Quigley and A. T. Broman, "The number of people with glaucoma worldwide in 2010 and 2020," British Journal of Ophthalmology, vol. 90, no. 3, pp. 262-267, 2006.

[9] V. B. Voleti and J.-P. Hubschman, "Age-related eye disease," Maturitas, vol. 75, no. 1, pp. 29-33, 2013.

[10] R. Vohra, J. C. Tsai, and M. Kolko, "The role of inflammation in the pathogenesis of glaucoma," Survey of Ophthalmology, vol. 58, no. 4, pp. 311-320, 2013.

[11] P. A. Williams, N. Marsh Armstrong, G. R. Howell et al., "Neuroinflammation in glaucoma: a new opportunity," Experimental Eye Research, vol. 157, pp. 20-27, 2017.

[12] B. E. K. Klein, R. Klein, and K. E. Lee, "Heritability of risk factors for primary open-angle glaucoma: the Beaver Dam Eye Study," Investigative Opthalmology \& Visual Science, vol. 45, no. 1, pp. 59-62, 2004.

[13] Y. Kim, B.-G. Han, and G. E. S. G. Ko, "Cohort profile: the Korean Genome and Epidemiology study (KoGES) consortium," International Journal of Epidemiology, vol. 46, no. 4, p. 1350, 2017.

[14] "Obesity: preventing and managing the global epidemic," pp. 1-253, 2000, Report of a WHO Consultation World Health Organization Technical Report Series 894 i-xii.

[15] E. Kelly, Q. Wen, D. Haddad, and J. O'Banion, "Effects of an aging population and racial demographics on eye disease prevalence: projections for Georgia through 2050," American Journal of Ophthalmology, vol. 210, pp. 35-40, 2020.

[16] B. A. Burt, "Periodontitis and aging: reviewing recent evidence," The Journal of the American Dental Association, vol. 125, no. 3, pp. 273-279, 1994.

[17] S. Renvert, R. E. Persson, and G. R. Persson, “Tooth loss and periodontitis in older individuals: results from the Swedish National Study on Aging and Care," Journal of Periodontology, vol. 84, no. 8, pp. 1134-1144, 2013.

[18] Y. Wu, G. Dong, W. Xiao et al., "Effect of aging on periodontal inflammation, microbial colonization, and disease susceptibility," Journal of Dental Research, vol. 95, no. 4, pp. 460-466, 2016.

[19] N.-Q. Huang, H. Jin, S.-y. Zhou, J.-s. Shi, and F. Jin, "TLR4 is a link between diabetes and Alzheimer's disease," Behavioural Brain Research, vol. 316, pp. 234-244, 2017.

[20] B. Song, Y. Zhang, L. Chen et al., "The role of Toll-like receptors in periodontitis," Oral Diseases, vol. 23, no. 2, pp. 168-180, 2017.

[21] L. V. Gankovskaya, N. M. Khelminskaya, E. A. Molchanova, and O. A. Svitich, "Role of innate immunity factors in periodontitis pathogenesis]," Zh Mikrobiol Epidemiol Immunobiol, vol. 100, p. 7, 2016.

[22] E. M. Cardoso, C. Reis, and M. C. Manzanares-Céspedes, "Chronic periodontitis, inflammatory cytokines, and interrelationship with other chronic diseases," Postgraduate Medicine, vol. 130, no. 1, pp. 98-104, 2018.

[23] R. N. Weinreb and P. T. Khaw, "Primary open-angle glaucoma," The Lancet, vol. 363, no. 9422, pp. 1711-1720, 2004.

[24] X. Wei, K.-S. Cho, E. F. Thee, M. J. Jager, and D. F. Chen, "Neuroinflammation and microglia in glaucoma: time for a paradigm shift," Journal of Neuroscience Research, vol. 97, no. 1, pp. 70-76, 2019.

[25] P. S. Kumar, "Oral microbiota and systemic disease," Anaerobe, vol. 24, pp. 90-93, 2013.

[26] B. Detrick and J. J. Hooks, "Immune regulation in the retina," Immunologic Research, vol. 47, no. 1-3, pp. 153-161, 2010.

[27] I. Soto and G. R. Howell, "The complex role of neuroinflammation in glaucoma," Cold Spring Harbor Perspectives in Medicine, vol. 4, 2014.

[28] B. Halliwell, "Reactive oxygen species in living systems: source, biochemistry, and role in human disease," The American Journal of Medicine, vol. 91, pp. 14S-22S, 1991.

[29] M. Kopáni, P. Celec, L. Danišovič, P. Michalka, and C. Biró, "Oxidative stress and electron spin resonance," Clinica Chimica Acta, vol. 364, no. 1-2, pp. 61-66, 2006.

[30] T. Zhang, O. Andrukhov, H. Haririan et al., "Total antioxidant capacity and total oxidant status in saliva of periodontitis patients in relation to bacterial load," Frontiers in Cellular and Infection Microbiology, vol. 5, p. 97, 2015.

[31] M. M. Huycke, V. Abrams, and D. R. Moore, "Enterococcus faecalis produces extracellular superoxide and hydrogen peroxide that damages colonic epithelial cell," DNA Carcinogenesis, vol. 23, pp. 529-536, 2002.

[32] B. Vlkova and P. Celec, "Does Enterococcus faecalis contribute to salivary thiobarbituric acid-reacting substances?" In Vivo, vol. 23, pp. 343-345, 2009.

[33] S. H. Byun, S. Lee, S. H. Kang, H. G. Choi, and S. J. Hong, "Cross-sectional analysis of the association between periodontitis and cardiovascular disease using the Korean Genome and Epidemiology study data," International Journal of Environmental Research and Public Health, vol. 17, no. 14, Article ID 5237, 2020. 
[34] S. H. Byun, C. Min, S. J. Hong, H. G. Choi, and D. H. Koh, "Analysis of the relation between periodontitis and chronic gastritis/peptic ulcer: a cross-sectional study using KoGES HEXA data," International Journal of Environmental Research and Public Health, vol. 17, no. 12, Article ID 4378, 2020.

[35] S. H. Byun, D. M. Yoo, J. W. Lee, and H. G. Choi, "Analyzing the association between hyperuricemia and periodontitis: a cross-sectional study using KoGES HEXA data," International Journal of Environmental Research and Public Health, vol. 17, no. 13, Article ID 4777, 2020.

[36] L. Shapira, R. Borinski, M. N. Sela, and A. Soskolne, "Superoxide formation and chemiluminescence of peripheral polymorphonuclear leukocytes in rapidly progressive periodontitis patients," Journal of Clinical Periodontology, vol. 18, no. 1, pp. 44-48, 1991.

[37] I. L. C. Chapple, "Reactive oxygen species and antioxidants in inflammatory diseases," Journal of Clinical Periodontology, vol. 24, no. 5, pp. 287-296, 1997.

[38] W. J. Loesche, J. P. Robinson, M. Flynn, J. L. Hudson, and R. E. Duque, "Reduced oxidative function in gingival crevicular neutrophils in periodontal disease," Infection and Immunity, vol. 56, no. 1, pp. 156-160, 1988.

[39] S. Kimura, T. Yonemura, and H. Kaya, "Increased oxidative product formation by peripheral blood polymorphonuclear leukocytes in human periodontal diseases," Journal of Periodontal Research, vol. 28, no. 3, pp. 197-203, 1993.

[40] D. Biasi, L. M. Bambara, A. Carletto et al., "Neutrophil migration, oxidative metabolism and adhesion in early onset periodontitis," Journal of Clinical Periodontology, vol. 26, no. 9, pp. 563-568, 1999.

[41] E. Niki, "Oxidative stress and antioxidants: distress or eustress?" Archives of Biochemistry and Biophysics, vol. 595, pp. 19-24, 2016.

[42] L. Tothova and P. Celec, "Oxidative stress and antioxidants in the diagnosis and therapy of periodontitis," Frontiers in Physiology, vol. 8, p. 1055, 2017.

[43] T. T. Nguyen, L. Q. Ngo, A. Promsudthi, and R. Surarit, "Salivary oxidative stress biomarkers in chronic periodontitis and acute coronary syndrome," Clinical Oral Investigations, vol. 21, no. 7, pp. 2345-2353, 2017.

[44] S. K. Myung, W. Ju, B. Cho et al., "Efficacy of vitamin and antioxidant supplements in prevention of cardiovascular disease: systematic review and meta-analysis of randomised controlled trials," BMJ, vol. 346, p. 10, 2013.

[45] U. Sezer, K. Erciyas, K. Üstün et al., "Effect of chronic periodontitis on oxidative status in patients with rheumatoid arthritis," Journal of Periodontology, vol. 84, no. 6, pp. 785-792, 2013.

[46] D. L. Williams, "Oxidative stress and the eye," Veterinary Clinics of North America: Small Animal Practice, vol. 38, no. 1, pp. 179-192, 2008.

[47] A. Spector, "Oxidative stress-induced cataract: mechanism of action," The FASEB Journal, vol. 9, no. 12, pp. 1173-1182, 1995.

[48] C. Erb and M. Heinke, "Oxidative stress in primary openangle glaucoma," Frontiers in Bioscience, vol. E3, no. 4, pp. 1524-1533, 2011.

[49] S. C. Saccà and A. Izzotti, "Oxidative stress and glaucoma: injury in the anterior segment of the eye," Progress in Brain Research, vol. 173, pp. 385-407, 2008.

[50] M. Paizan and J. Vilela Martin, "Is there an association between periodontitis and hypertension?" Current Cardiology Reviews, vol. 10, no. 4, pp. 355-361, 2014.
[51] A. Khatri, J. K. Shrestha, M. Thapa, B. K. Khatri, and M. Kharel, "Severity of primary open-angle glaucoma in patients with hypertension and diabetes," Diabetes, Metabolic Syndrome and Obesity: Targets and Therapy, vol. 11, pp. 209-215, 2018.

[52] P. A. Newman Casey, N. Talwar, B. Nan, D. C. Musch, and J. D. Stein, "The relationship between components of metabolic syndrome and open-angle glaucoma," Ophthalmology, vol. 118, pp. 1318-1326, 2011.

[53] B. G. Loos, J. Craandijk, F. J. Hoek, P. M. E. W.-v. Dillen, and U. V. D. Velden, "Elevation of systemic markers related to cardiovascular diseases in the peripheral blood of periodontitis patients," Journal of Periodontology, vol. 71, no. 10, pp. 1528-1534, 2000.

[54] C. Benguigui, V. Bongard, J.-B. Ruidavets et al., "Metabolic syndrome, insulin resistance, and periodontitis: a cross-sectional study in a middle-aged French population," Journal of Clinical Periodontology, vol. 37, no. 7, pp. 601-608, 2010. 